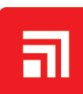 \\ CHITKARA \\ Journal of Nuclear Physics, Material Sciences, Radiation and Applications \\ Journal homepage: https://jnp.chitkara.edu.in/
}

\section{Time Resolution Measurements on SiPM for High Energy Physics Experiments}

L. M. Montano ${ }^{1}$ and M. Fontaine ${ }^{1 *}$

${ }^{1} I P N$ Research and Advanced Studies Center, cdmx

"Email: mfontaine@fis.cinvestav.mx

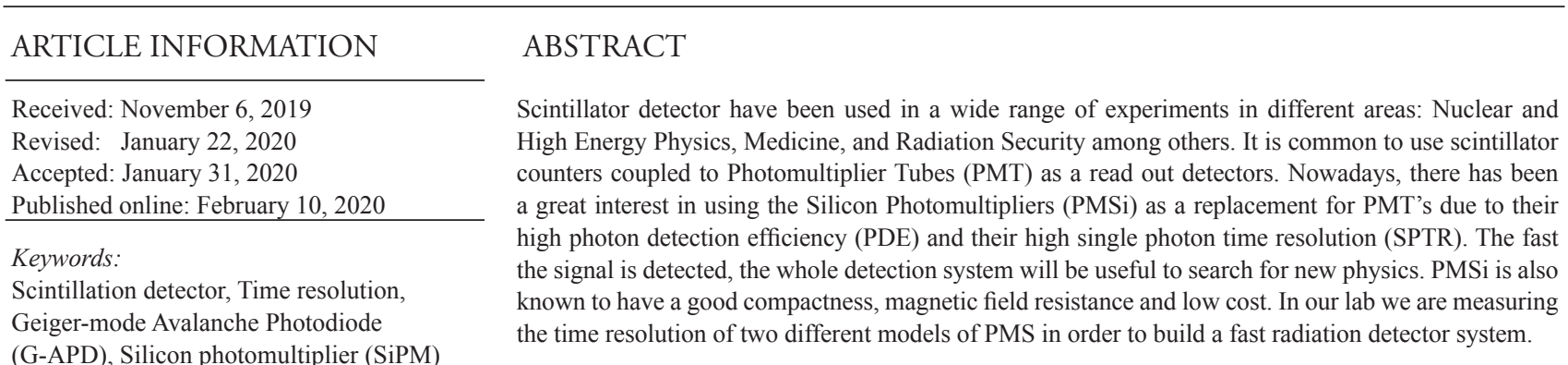

\section{Introduction}

In High Energy Physics, scintillation detectors is used commonly to detect charged particles. These detectors basically consist of a scintillation material that can be liquid or solid; these last can be plastic or crystal scintillations, which generate a light when charged particles hit it or by high-energy electromagnetic waves interactions. Scintillators are coupled with light sensors that detect the light generated in the plastic. These sensors previously were the photomultiplier tubes. Nowadays, the use of solid-state photomultipliers ( $\mathrm{SiPM}$ ) is being generalized in addition to an associated electronics, which is very precise and accurate for data acquisition, processing and analysis.

\section{Description}

In the present paper, we describe the objective, the procedure and the results of the characterization of the low level light sensors called SiPM (Silicon photomultipliers), as well as the characterization, description and comparative analysis of 4 types of plastic scintillators. Finally, there was the choice of the elements to make the most efficient scintillation detector to detect charged high energy particles.

\section{Plastic Scintillator Selection}

Four different kind of plastic scintillators were tested. The plastic which collected more light was chosen coupled with the same SiPM. BC-404 was the most efficient detector in this case, see Figure 1.

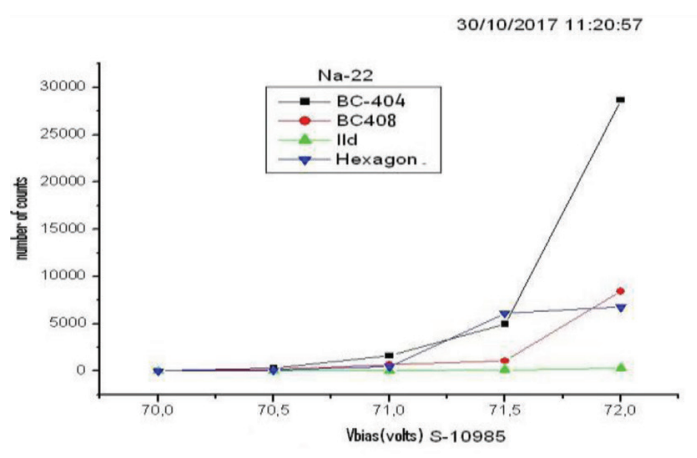

Figure 1: Comparison among four kind of plastic scintillators.

\section{Characterization of SiPM}

The SiPM are light sensors (in this case scintillator light) made of thousands of photodiodes (APD) connected in parallel in order to accumulate all the signals for a final magnified one.

Taking into account the electronic specifications, the Hamamatsu SiPM brand was selected over the SensL SiPM brand (see Table 1). Its characterization was done as follows.

Calculate and design the polarization circuit of the SiPM, Determine its Quantum Efficiency, Photon Detection Efficiency (PDE) of both SiPM, and measure its Dark Current. 
Specifications comparison sheet, Hamamatsu \& SensL SiPM

Table 1: Comparison of the specifications for 2 different SiPM devices from different manufacturers: Hamamatsu Brand Type S10985 and Brand SensL Type C 60035 [1].

\begin{tabular}{|c|c|c|c|c|}
\hline Parameter & Symbol & Hamamatsu & SensL & Units \\
\hline Number of channels & & $4(2 X 2)$ & $4(2 \times 2)$ & ch \\
\hline Effective area / channel & & $3 \times 3$ & $6 \times 6$ & $\mathrm{~mm}$ \\
\hline Number of pixels / channel & & 3600 & 18980 & p-ch \\
\hline Pixel size & & $50 \times 50$ & 35 & um \\
\hline Factor fill & & 61.5 & 64 & $\%$ \\
\hline Spectral response range & $\lambda$ & 320 a 900 & 300950 & $\mathrm{~nm}$ \\
\hline Sensitive peak wavelength & $\lambda \mathrm{p}$ & 440 & 420 & $\mathrm{~nm}$ \\
\hline Operating voltage range & & $70+/-10$ & $24.5+5$ & $\mathrm{~V}$ \\
\hline Dark counts / channel & & 6000 & 1200 & kcps \\
\hline $\begin{array}{l}\text { Maximum dark count / } \\
\text { channel }\end{array}$ & & 10000 & 3400 & kcps \\
\hline $\begin{array}{l}\text { Terminal / channel } \\
\text { capacitance }\end{array}$ & $\mathrm{Ct}$ & 320 & 48 & $\mathrm{pF}$ \\
\hline Gain & $\mathrm{M}$ & $7.5 \times 10^{*} 5$ & $3 \times 10^{*} 6$ & - \\
\hline PDE at $\lambda p$ & & 50 & 41 & $\%$ \\
\hline
\end{tabular}
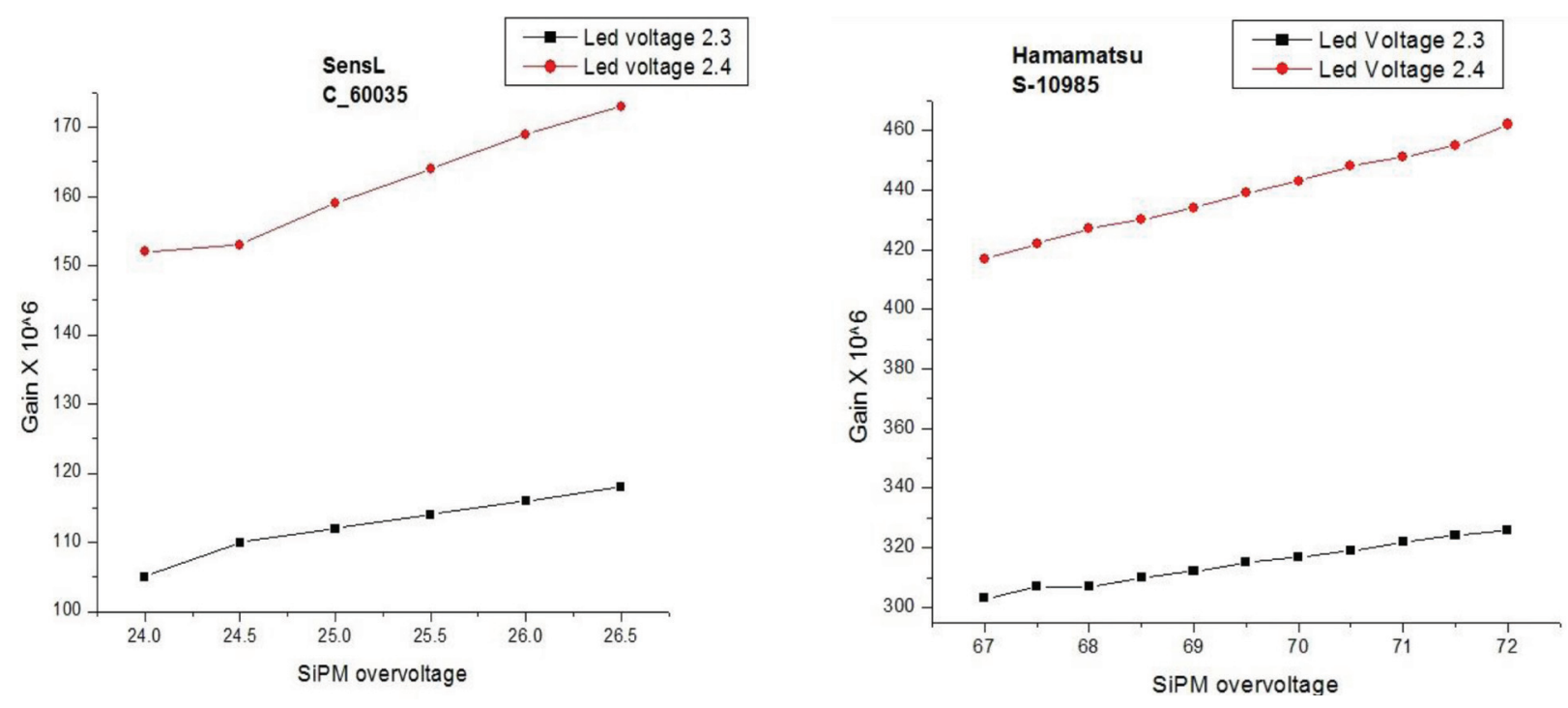

Figure 2: Gain comparison between SiPM's.

Note: All tests were performed at room temperature.

\section{a) SiPM Polarization Circuit}

In order to increase the temporal resolution, the decay and recovery time was reduced, modifying the polarization circuit of the SiPM.

\section{b) Calculate your Quantum Efficiency}

To obtain the first photon detection efficiency (PDE); quantum efficiency of the SiPM was calculated, for which the following procedure was performed: (i) The level of illumination (Illuminance) generated by the blue LED (460ns) was measured by means of a calibrated Luxometer.

(ii) The amount of light (Luxes) in power (Watts) was converted, using the formula

$$
P(W)=E v(l x) \times A\left(m^{2}\right) / \eta(l m / W)
$$

$P(W)=0.4 L x \times 0.0000785 m^{2} / 60=5.23 e^{-7 W}$ 
Where $\mathrm{P}=$ Power in Watts (W), is equal to the illuminance Ev in lux (lx) multiplied by the surface area $A$ in square meters $\left(\mathrm{m}^{2}\right)$, divided by the luminous efficiency $\eta$ in lumens per watt $(\mathrm{lm} / \mathrm{W})$ :

(iii) The current of the SiPM was measured in picoAmpers and the following formula was applied

$$
Q E=\frac{\text { Photocurrent Multiplicated }}{\text { Phocurrent Before Multiplication }}
$$

$$
\begin{aligned}
Q E & =\frac{1 m A}{4.65 u A}=215 E Q(S 10985) \\
& =215 \% E Q(C 60035)=162 \%
\end{aligned}
$$

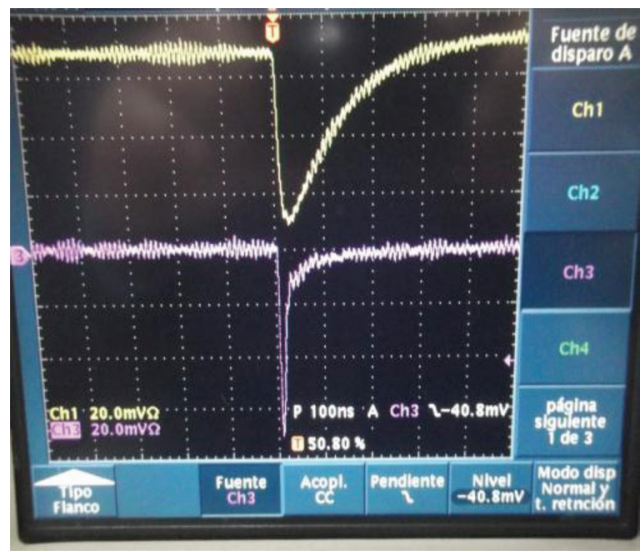

Figure 3: Polarization circuit before (up) and after (down) electronic improvement.

\section{c) Determine the Photon Detection Efficiency (PDE)}

Fourth, the PDE was determined by the following formula. $\mathrm{PDE}=\mathrm{Fg} \times \mathrm{QE} \times \mathrm{Pa}$

Where: Fg = Fill factor, $\mathrm{QE}=$ quantum efficiency, $\mathrm{Pa}=$ avalanche probability

$\mathrm{PDE}=0.65 \times 215 \times 0.1=26.23 \%$

\section{d) Dark Current}

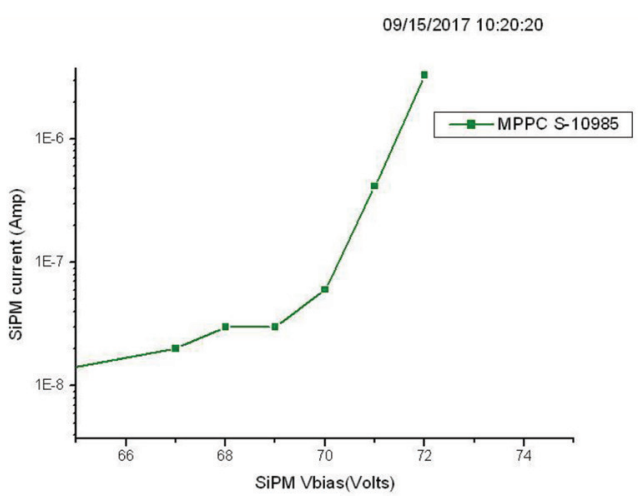

(a)

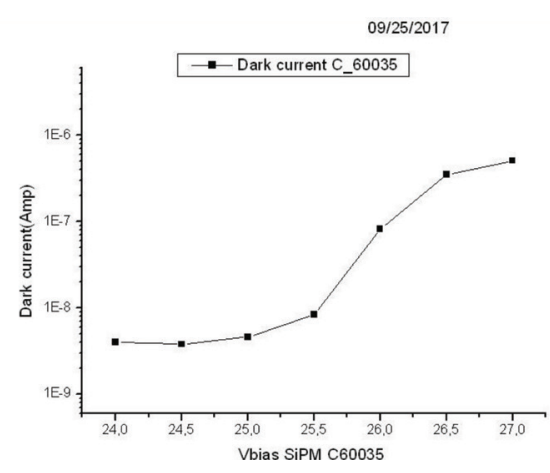

(b)

Figure 4: Comparison of dark currents of both SiPM.

\section{TDC Calibration}

To measure the temporal resolution of the SiPM scintillation detector and BC 404 plastic, first a system calibration was done (Figure 5) and TDC module of type VME, V792N was used. The data was acquired on the computer through a CAEN V1718 interface module and through a Labview program. See Figure 6.

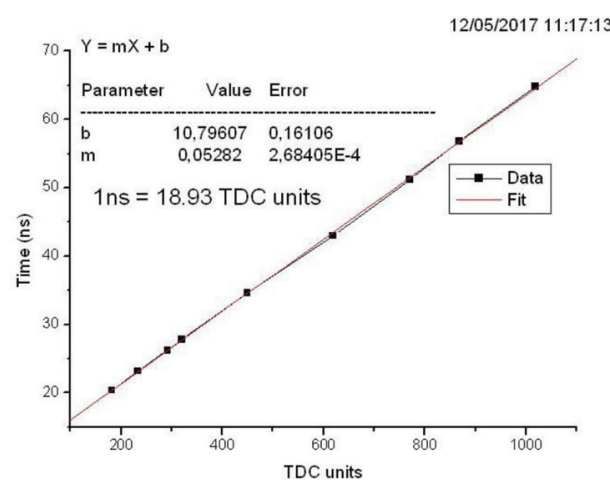

Figure 5: TDC calibration.

\section{Obtain an Approximation of the Temporal Resolution}

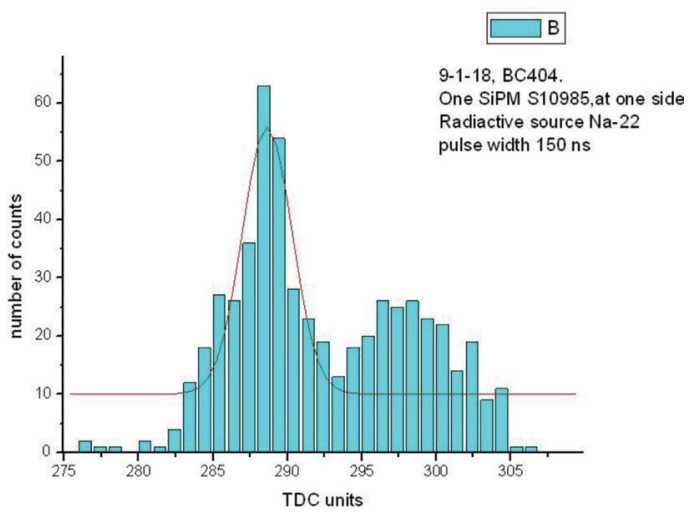

Figure 6: TDC temporal resolution. 


\section{Results}

Among the main results are the reduction of the pulse width of the SiPM from 300 ns to less than 100 ns and the decay time from 20 to $5 \mathrm{~ns}$; it was possible to find the quantum efficiency of the two SiPM and a comparative analysis of the four types of scintillator plastic and the best elements were chosen to elaborate the Detector and characterize it; also the TDC system was calibrated and its temporal resolution was measured.

Pending work or next steps, find the correlation between the time and the load of the signals to be able to calculate the temporal resolution based on the energy. For this, it is necessary to be able to work with the TDC and the QDC simultaneously.

\section{References}

[1] J-Series High PDE and Timing Resolution, TSV Package USER MANUAL, SensL.
[2] J. W. Zhao et al., Nuclear Instruments and Methods in Physics Research Section A, Accelerators Spectrometers Detectors and Associated Equipment 823, 41 (2016). https://doi.org/10.1016/j.nima.2016.03.106.

[3] P. W. Cattaneo et al., IEEE Trans. Nucl. Sci. 61, 2657 (2014). https://doi.org/10.1109/TNS.2014.2347576

[4] P. Eckert et al., Nuclear Instruments and Methods in Physics Research Section A: Accelerators, Spectrometers, Detectors and Associated Equipment 620, 217 (2010). https://doi.org/10.1016/j.nima.2010.03.169

[5] G. F. Knoll, Radiation Detection and Measurement, Wiley, India (2010).

[6] W. R. Leo, Techniques for Nuclear and Particle Physics Experiments: A How-To Approach, Springer-Verlag Berlin Heidelberg (1994). 


\section{Journal of Nuclear Physics, Material Sciences, Radiation and Applications}

Chitkara University, Saraswati Kendra, SCO 160-161, Sector 9-C, Chandigarh, 160009, India

Volume 7, Issue 1

August 2019

ISSN 2321-8649

Copyright: [C 2019 L. M. Montano and M. Fontaine] This is an Open Access article published in Journal of Nuclear Physics, Material Sciences, Radiation and Applications (J. Nucl. Phy. Mat. Sci. Rad. A.) by Chitkara University Publications. It is published with a Creative Commons Attribution- CC-BY 4.0 International License. This license permits unrestricted use, distribution, and reproduction in any medium, provided the original author and source are credited. 